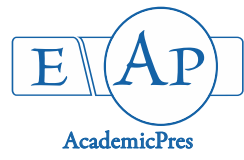

\title{
Phytogeographical Affinities of Tree Species of Similipal Biosphere Reserve, Odisha, India
}

\author{
Manas R. MOHANTA, Anil K. BISWAL, Sudam C. SAHU* \\ North Orissa University, Department of Botany, Baripada 757003, India; sudamsahu.bdk@gmail.com (" ${ }^{*}$ corresponding author)
}

\begin{abstract}
The phytogeography of Similipal Biosphere Reserve (SBR), Odisha, India, reveals very interesting information on distribution of tree species. Phytogeographical affinities of tree species of SBR has been analysed by obtaining the information about the species distribution at local and global scale. A total of 240 tree species were recorded and their phytogeographical affinities were compiled with different countries of the globe. An analysis of the affinities revealed that SBR has strong affinity with Sri-Lanka (46.66\%) and Myanmar (45.83\%) followed by China, Malaysia, Thailand, Australia and Africa. SBR has also affinity with Himalayan vegetation possessing several trees and orchids find distribution in both the areas. The phytogeographical affinity of SBR supports the migration, establishment and naturalization of flora from/to SBR. This hypothesis needs further study for biogeographical mapping of Indian sub-continent.
\end{abstract}

Keywords: Biogeographical mapping; conservation of species; distribution of trees; ecological implications; Indian subcontinent

Abbreviations: SBR - Similipal Biosphere Reserve

\section{Introduction}

In recent time, remarkable changes have been observed in the environment due to both man-made and natural factors resulting destruction and degradation of natural habitats. Biodiversity loss is a global phenomenon but its impact is greatest in the tropics, where the majority of species are distributed (Collen et al., 2008). Tropical forests harbour ca $50 \%$ of total species in the world occupying only $7 \%$ land area which is getting disappeared at a rate of 0.8 to $2 \%$ per year (Sagar et al., 2003). The phytogeographical studies are the useful tools for assessment of the geographical distribution, origin and ecological implications of plants of an area (Lausi and Nimis, 1985).

India is a mega biodiversity country where the floral distribution and diversity is highly influenced by its varied climatic, topographic and edaphic conditions. Starting from the north Himalayas to the south Nilgiri hills and Western Ghats a wide range of diversity in flora and their habitat is observed. According to great British botanist Sir JD Hooker (1904), "the Indian flora is more varied than any of the country of equal area in the eastern hemisphere, if not in the globe". Similarly the state of Odisha in Eastern India is rich in diversity and inhabited mixture of north and south Indian flora. JS Gamble (1892) was to comment that Orissa is the meeting ground for the Himalayan and the South Indian Elements of the Indian flora. The available literature also shows significant affinity of Indian floral with other parts of the world. The floristic elements of India share the predominant affinities with the Indo-Malayan elements. It has also affinities with Afro-Tropical and Tropical- Asian elements (Daniel and Nair, 1986). Many workers have contributed moderately on phytogeographical study of Indian sub-continent. Rana et al. (2001) studied the phytogeographical affinity of Tons valley, Uttaranchal; Suresh and Sukumar (1999) on Nilgiri Biosphere Reserve and Pragason (2014) on Eastern Ghats, Sukumar et al. (1992) on Mudumalai reserve forest; Saravanan et al. (2013) on Andaman etc. Globally, studies on phytogeography of several regions are available such as Neotropics (Gentry, 1982), Friulian plain, NE Italy (Nimis and Fonda, 1997), Maxico (Rzedowski,1975)Western Europe and Eastern Greenland (Hubbard and Boulter, 2000), Tasmanian alpine flora (Kirkpatrick, 1982), Sonoran and Chihuahuan deserts (Krings, 2000), Yukon territory, NW Canada (Lausi and Nimis, 1985), Yucatan Peninsula (Manrique et al., 2003), arid mountain of Oman (Ghazanfar, 1991) and Coastal vegetation of Yucatan Peninsula (Espejel, 1987).

Phytogeographical study is useful in many aspects like conservation of species, sustainable utilization resources, study of migration of species and speciation, study on phenology and adaptation etc. Based on the phytogeographical data several postulations have been given regarding migration of flora in Indian subcontinent. Takhtajan, (1969) postulated that the angiosperms spread 
from low to high altitudes and not in the opposite direction and Razi, (1955) felt that the vegetation existed in South India comprising Eastern Ghats much before the advent of Himalayas and Similipal was part of Gondwana land mass in the Paleozoic era. Being the meeting point of the northern and southern flora, it is clear that SBR has major role in the migration of flora within Indian subcontinent. Some bio geographers consider Similipal Hill ranges as part of Eastern Ghats of India and some other put the region under Chotanagpur plateau. However, SBR in Odisha is very rich in floral diversity and a grand repository of many rare, endemic and threatened species. But, a comprehensive account of phytogeographical affinity of taxa is lacking. The present study focuses on phytogeographical affinity of tree species of SBR and its significance.

\section{Materials and Methods}

\section{Studyarea}

Similipal Biosphere Reserve (SBR) in Odisha is very rich in floral diversity and a grand repository of many rare, endemic and threatened species. It is situated between $21^{\circ}$ $28^{\prime}$ to $22^{\circ} 08^{\prime} \mathrm{N}$ latitude and $86^{\circ} 03^{\prime}$ to $86^{\circ} 37^{\prime} \mathrm{E}$ longitude. SBR exhibits a great degree of topographic variation and thus provides adobe for rich biodiversity (Mishra, 2010). Saxena and Brahmam (1989) categorised SBR into 6 major forest types are Northern tropical moist deciduous forests, Northern tropical semi-evergreen forests, Dry deciduous hill forest, Dry sal forests, High level Sal forests and Grasslands. There are 1076 plants recorded from the area including 60 species of ferns, 92 species of orchids and two gymnosperms (Saxena and Brahmam, 1989; Misra, 2004).The climate is warm and humid with an average rainfall of $173 \mathrm{~cm}$ (maximum $225 \mathrm{~cm}$ and minimum $110 \mathrm{~cm}$ ) and maximum temperature during hot weather rise up to 43 degree Celsius while minimum during winter fall as low as 4 degree Celsius (Mishra, 2010).
Extensive field studies were conducted during different seasons of the year in order to access floristic composition of the study area (Fig. 1). Collection of plant samples along with their photographs were made for future references. Collected plant specimens were identified referring regional floras. All the identified plants were processed and deposited at Herbarium of Department of Botany, North Orissa University. A list of tree species had been made and categorised into various phytogeographical groups according to their world distribution (Table 1). The countries considered for the phytogeographical study were Sri-Lanka, Myanmar, China, Thailand, Malaysia, Australia and Africa. The phytogeographical affinity was obtained from in consultation with floras like flora of Madhya Pradesh (Verma et al., 1993), flora of Odisha (Saxena and Brahmam, 1994-1996) and flora of Bihar (Singh et al., 2001 ), floral diversity of Similipal Biosphere Reserve, Vol-1 (Biswal et al., 2011) and trees of Similipal Biosphere Reserve (Nayak et al., 2014).

\section{Results and Discussion}

The affinity of tree flora of SBR with other subcontinents is very extensive. The result shows highest similarity of tree species with Sri- Lanka and least with Africa (Fig. 2). The affinity of SBR with Sri- Lanka is found to be $46.66 \%$, Myanmar $45.83 \%$, China $25.00 \%$, Thailand and Malaysia $12.5 \%$ each, Australia $7.5 \%$ and Africa $4.58 \%$. All the members of Ebenaceae found in SBR are distributed in Sri- Lanka and some of the members of Moraceae are very similar to Sri- Lanka. Albizia chinensis, Albizia odoratissima, Carallia branchiate, Cipadessa baccifera etc. are the plants with highest range of distributions among countries. IndoAfrican and Indo-Australlian affinity found to be very negligible; only 11 and 17 species are found to be common respectively.

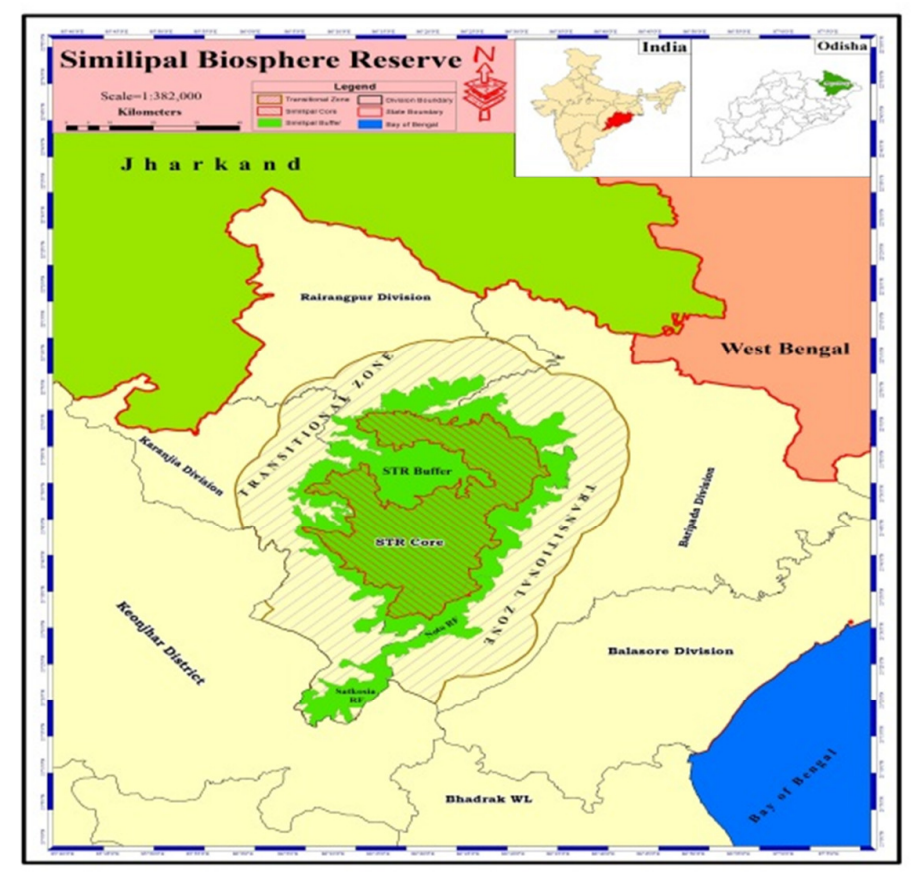

Fig. 1. Map of the study area 
356

Table 1 . List of 240 tree species of SBR and their affinity

\begin{tabular}{|c|c|c|c|c|c|c|c|c|c|}
\hline Sl. No & Plant Name & Family & A & B & $\mathrm{C}$ & $\mathrm{D}$ & $\mathrm{E}$ & $\mathrm{F}$ & $\bar{G}$ \\
\hline 1 & Acacia farnesiana & Leguminosae & - & - & - & - & - & - & - \\
\hline 2 & Acacia lenticularis & Leguminosae & - & - & - & - & - & - & - \\
\hline 3 & Acacia nilotica & Leguminosae & - & - & - & - & - & - & - \\
\hline 4 & Acronychia pedunculata & Rutaceae & + & - & + & - & + & + & - \\
\hline 5 & Actinodaphne gullavara & Lauraceae & - & - & + & - & - & - & - \\
\hline 6 & Aegle marmelos & Rutaceae & - & - & - & - & - & - & - \\
\hline 7 & Alangium salvifolium & Cornaceae & + & + & - & + & - & - & + \\
\hline 8 & Albizia procera & Fabaceae & + & - & + & - & + & - & - \\
\hline 9 & Albizia chinensis & Fabaceae & + & + & + & + & + & - & - \\
\hline 10 & Albizia lebbeck & Fabaceae & - & - & - & - & - & - & + \\
\hline 11 & Albizia odoratissima & Fabaceae & + & + & + & + & + & - & - \\
\hline 12 & Alchornea mollis & Euphorbiaceae & - & - & - & - & - & - & - \\
\hline 13 & Alphonsea lutea & Annonaceae & + & - & - & - & - & - & - \\
\hline 14 & Alphonsea ventricosa & Annonaceae & - & - & - & - & - & - & - \\
\hline 15 & Alstonia scholaris & Apocynaceae & + & + & - & - & + & - & + \\
\hline 16 & Annona reticulate & Annonaceae & - & - & - & - & - & - & - \\
\hline 17 & Annona squamosa & Annonaceae & - & - & - & - & - & - & - \\
\hline 18 & Anogeissus acuminate & Combretaceae & - & - & + & - & - & - & - \\
\hline 19 & Anogeissus latifolia & Combretaceae & + & - & - & - & - & - & - \\
\hline 20 & Antidesma acidum & Phyllanthaceae & - & + & + & + & - & - & - \\
\hline 21 & Antidesma bunius & Phyllanthaceae & + & - & + & - & - & - & - \\
\hline 22 & Antidesma ghaesembilla & Phyllanthaceae & + & + & - & - & - & + & - \\
\hline 23 & Antidesma montanum & Phyllanthaceae & + & + & + & - & - & + & + \\
\hline 24 & Aphanamixis polystachya & Meliaceae & + & - & + & - & - & - & - \\
\hline 25 & Aporosa octandra & Phyllanthaceae & - & - & + & - & - & - & - \\
\hline 26 & Ardisia solanacea & Primulaceae & + & + & + & - & - & - & - \\
\hline 27 & Artocarpus beterophyllus & Moraceae & - & - & - & - & - & - & - \\
\hline 28 & Artocarpus lacucha & Moraceae & - & - & - & - & - & - & - \\
\hline 29 & Atlantia monophylla & Rutaceae & + & - & + & - & - & - & - \\
\hline 30 & Azadirachta indica & Meliaceae & - & + & + & - & - & - & - \\
\hline 31 & Baccaurea ramiflora & Phyllanthaceae & - & - & + & - & - & - & - \\
\hline 32 & Barringtonia acutangula & Lecythidaceae & - & - & - & - & - & + & - \\
\hline 33 & Baubinia purpurea & Fabaceae & - & - & - & - & - & - & - \\
\hline 34 & Baubinia semla & Fabaceae & - & - & - & - & - & - & - \\
\hline 35 & Bauhinia variegata & Fabaceae & - & + & + & - & - & - & - \\
\hline 36 & Baubinia malabarica & Fabaceae & - & - & + & - & - & - & - \\
\hline 37 & Benkara fasciculata & Rubiaceae & + & - & - & - & - & - & - \\
\hline 38 & Bischofia javanica & Phyllanthaceae & - & - & + & - & - & - & - \\
\hline 39 & Bombax ceiba & Malvaceae & - & - & - & - & - & - & - \\
\hline 40 & Boswellia serrata & Burseraceae & - & - & - & - & - & - & - \\
\hline 41 & Bridelia glauca & Phyllanthaceae & - & - & + & - & - & - & - \\
\hline 42 & Bridelia retusa & Phyllanthaceae & + & - & + & - & - & - & - \\
\hline 43 & Buchanania cochinchinensis & Anacardiaceae & - & - & + & - & - & - & - \\
\hline 44 & Butea monosperma & Fabaceae & - & - & - & - & + & - & - \\
\hline 45 & Callicarpa tomentosa & Lamiaceae & + & - & + & + & - & - & - \\
\hline 46 & Canthium glabrum & Rubiaceae & - & - & + & - & - & - & - \\
\hline 47 & Carallia branchiata & Rhizophoraceae & + & + & + & + & + & + & - \\
\hline 48 & Careya arborea & Lecythidaceae & - & - & - & - & - & - & - \\
\hline 49 & Casearia graveolens & Salicaceae & - & - & + & - & - & - & - \\
\hline 50 & Casearia tomentosa & Salicaceae & + & - & - & - & - & + & - \\
\hline 51 & Cassia fistula & Fabaceae & + & + & + & - & - & - & - \\
\hline 52 & Cassine glauca & Celastraceae & + & + & - & + & - & - & - \\
\hline 53 & Catunaregam spinosa & Rubiaceae & + & - & - & - & - & - & - \\
\hline 54 & Celtis tetrandra & Cannabaceae & - & - & - & + & - & - & - \\
\hline 55 & Chionanthus mala-elengi & Oleaceae & - & - & - & - & - & - & - \\
\hline 56 & Chionanthus ramiflorus & Oleaceae & - & + & + & - & + & - & - \\
\hline 57 & Chloroxylon swietenia & Rutaceae & + & - & - & - & - & - & - \\
\hline 58 & Cipadessa baccifera & Meliaceae & + & + & + & + & + & - & - \\
\hline 59 & Cleistanthus collinus & Phyllanthaceae & + & - & - & - & - & - & - \\
\hline 60 & Cleistanthuspatulus & Phyllanthaceae & + & - & - & - & - & - & - \\
\hline
\end{tabular}




\begin{tabular}{|c|c|c|c|c|c|c|c|c|c|}
\hline 61 & Cochlospermum religiosum & Bixaceae & + & - & + & - & + & - & - \\
\hline 62 & Cordia dichotoma & Boranginaceae & + & - & - & - & - & - & + \\
\hline 63 & Cordia macleodii & Boranginaceae & - & - & - & - & - & - & - \\
\hline 64 & Cordia monoica & Boranginaceae & + & - & - & - & - & - & - \\
\hline 65 & Crateva marmelos & Capparaceae & + & + & + & - & - & - & - \\
\hline 66 & Crateva religiosa & Capparaceae & + & - & + & - & - & - & - \\
\hline 67 & Croton persimilis & Euphorbiaceae & + & - & + & - & - & - & - \\
\hline 68 & Dalbergia lanceolaria & Leguminosae & + & - & + & - & - & - & - \\
\hline 69 & Dalbergia latifolia & Leguminosae & - & + & - & - & - & - & - \\
\hline 70 & Desmodium oojeinense & Leguminosae & - & - & - & - & - & - & - \\
\hline 71 & Dillenia aurea & Dilleniaceae & - & - & + & + & - & - & - \\
\hline 72 & Dillenia indica & Dilleniaceae & - & + & + & + & - & - & - \\
\hline 73 & Dillenia pentagyna & Dilleniaceae & - & + & + & + & - & - & - \\
\hline 74 & Dimorphocalyx glabellus & Euphorbiaceae & + & - & - & - & - & - & - \\
\hline 75 & Diospyros malabarica & Ebenaceae & + & + & - & - & + & - & - \\
\hline 76 & Diospyros melanoxylon & Ebenaceae & + & - & - & - & - & - & - \\
\hline 77 & Diospyros Montana & Ebenaceae & + & - & - & - & + & + & - \\
\hline 78 & Diospyros sylvatica & Ebenaceae & + & - & - & - & - & - & - \\
\hline 79 & Diospyros vera & Ebenaceae & + & - & + & - & - & - & - \\
\hline 80 & Ehretia acuminata & Boranginaceae & - & - & + & - & - & - & - \\
\hline 81 & Ehretia laevis & Boranginaceae & - & - & - & - & - & - & - \\
\hline 82 & Elaeocarpusstipularis & Elaeocarpaceae & - & - & - & - & - & - & - \\
\hline 83 & Elaeocarpus tectorius & Elaeocarpaceae & + & + & - & - & + & - & - \\
\hline 84 & Embelia ribes & Primulaceae & + & + & - & - & - & - & - \\
\hline 85 & Embelia tsjeriam-cottam & Primulaceae & + & - & - & - & - & - & - \\
\hline 86 & Erythrina suberosa & Leguminosae & - & - & + & + & - & - & - \\
\hline 87 & Euonymus glaber & Celastraceae & - & - & - & - & - & - & - \\
\hline 88 & Falconeria insignis & Euphorbiaceae & + & + & + & - & - & - & - \\
\hline 89 & Ficus benghalensis & Moraceae & + & - & - & - & - & - & - \\
\hline 90 & Ficus benjamina & Moraceae & + & - & + & - & - & - & - \\
\hline 91 & Ficus hispida & Moraceae & + & + & - & - & - & - & - \\
\hline 92 & Ficus microcarpa & Moraceae & + & + & + & - & - & - & - \\
\hline 93 & Ficus nervosa & Moraceae & + & + & + & - & - & - & - \\
\hline 94 & Ficus racemosa & Moraceae & + & + & - & - & - & - & - \\
\hline 95 & Ficus religiosa & Moraceae & + & - & + & + & - & - & - \\
\hline 96 & Ficus rumphii & Moraceae & - & - & + & - & - & - & - \\
\hline 97 & Ficus semicordata & Moraceae & - & - & + & - & - & - & - \\
\hline 98 & Ficus virens & Moraceae & + & + & + & - & - & - & - \\
\hline 99 & Ficus exasperata & Moraceae & + & - & - & - & - & - & + \\
\hline 100 & Firmiana colorata & Malvaceae & - & - & - & - & - & - & - \\
\hline 101 & Firmiana simplex & Malvaceae & + & - & - & - & - & - & - \\
\hline 102 & Flacourtia indica & Salicaceae & - & - & - & - & - & - & + \\
\hline 103 & Flacourtia jangomas & Salicaceae & - & - & - & - & - & - & - \\
\hline 104 & Flueggea virosa & Phyllanthaceae & + & - & - & - & - & + & + \\
\hline 105 & Garcinia cowa & Clusiaceae & - & - & + & + & - & - & - \\
\hline 106 & Garcinia xanthochymus & Clusiaceae & - & - & + & + & - & - & - \\
\hline 107 & Gardenia latifolia & Rubiaceae & + & - & - & - & - & - & - \\
\hline 108 & Garuga pinnata & Burseraceae & - & + & + & - & - & - & - \\
\hline 109 & Glochidion beyneanum & Phyllanthaceae & - & - & + & - & - & - & - \\
\hline 110 & Glocbidion lanceolarium & Phyllanthaceae & - & - & + & - & - & - & - \\
\hline 111 & Glochidion zeylanicum & Phyllanthaceae & + & - & - & - & - & - & - \\
\hline 112 & Gmelina arborea & Lamiaceae & - & - & - & - & - & - & - \\
\hline 113 & Grewia eriocarpa & Malvaceae & + & - & + & - & - & - & - \\
\hline 114 & Grewia orbiculata & Malvaceae & - & - & - & - & - & - & - \\
\hline 115 & Grewia serrulata & Malvaceae & - & - & - & - & - & - & - \\
\hline 116 & Grewia tiliifolia & Malvaceae & + & - & + & - & - & - & + \\
\hline 117 & Guazuma ulmifolia & Malvaceae & - & - & + & - & + & - & - \\
\hline 118 & Holarrhena pubescens & Apocynaceae & - & + & + & - & - & - & - \\
\hline 119 & Haldina cordifolia & Rubiaceae & + & + & - & + & - & - & - \\
\hline 120 & Heynea trijuga & Meliaceae & - & - & + & - & - & - & - \\
\hline 121 & Holoptelea integrifolia & Ulmaceae & + & + & - & - & - & - & - \\
\hline 122 & Homalium napaulense & Saliaceae & - & - & - & - & - & - & - \\
\hline
\end{tabular}


Mohanta MR et al / Not Sci Biol, 2018, 10(3):354-362

358

\begin{tabular}{|c|c|c|c|c|c|c|c|c|c|}
\hline 123 & Hymenodictyon orixense & Rubiaceae & - & - & + & - & - & - & - \\
\hline 124 & Ixora pavetta & Rubiaceae & + & - & + & - & - & - & - \\
\hline 125 & Kydia calycina & Malvaceae & - & + & + & - & - & - & - \\
\hline 126 & Lagerstroemia parviflora & Lythraceae & - & - & - & - & - & - & - \\
\hline 127 & Lagerstroemia speciosa & Lythraceae & + & + & + & - & - & - & - \\
\hline 128 & Lannea coromandelica & Anacardiaceae & - & - & - & - & + & - & - \\
\hline 129 & Lasiococca comberi & Euphorbiaceae & - & - & - & - & - & - & - \\
\hline 130 & Lepisanthes rubiginosa & Sapindaceae & - & - & - & - & - & + & - \\
\hline 131 & Ligustrum gamblei & Oleaceae & - & - & - & - & - & - & - \\
\hline 132 & Litseaglutinosa & Lauraceae & + & + & + & - & + & - & - \\
\hline 133 & Litsea monopetala & Lauraceae & - & + & + & - & - & - & - \\
\hline 134 & Litsea nitida & Lauraceae & - & - & + & - & - & - & - \\
\hline 135 & Macaranga denticulata & Euphorbiaceae & - & - & + & - & - & - & - \\
\hline 136 & Macaranga peltata & Euphorbiaceae & - & - & - & - & - & - & - \\
\hline 137 & Madhuca longifolia & Sapotaceae & + & - & + & - & - & - & - \\
\hline 138 & Magnolia champaca & Magnoliaceae & - & + & + & + & - & - & - \\
\hline 139 & Mallotus nudiflorus & Euphorbiaceae & + & + & + & - & - & - & - \\
\hline 140 & Mallotus philippensis & Euphorbiaceae & + & + & + & - & - & + & - \\
\hline 141 & Mangifera indica & Anacardiaceae & - & - & - & - & - & - & - \\
\hline 142 & Maytenus bailadillana & Celastraceae & - & - & - & - & - & - & - \\
\hline 143 & Melia azedarach & Meliaceae & - & + & + & - & - & - & - \\
\hline 144 & Meliosma pinnata & Sabiaceae & - & - & + & - & - & - & - \\
\hline 145 & Meliosma simplicifolia & Sabiaceae & + & + & + & - & - & - & - \\
\hline 146 & Memecylon umbellatum & Melastomataceae & + & - & + & + & - & - & - \\
\hline 147 & Mesua ferrea & Calophyllaceae & - & - & - & - & - & - & - \\
\hline 148 & Meynaspinosa & Rubiaceae & - & - & + & - & - & - & - \\
\hline 149 & Micromelum minutum & Rutaceae & + & - & - & - & + & - & - \\
\hline 150 & Miliusa tomentosa & Annonaceae & - & - & - & - & - & - & - \\
\hline 151 & Miliusa velutina & Annonaceae & - & - & - & - & - & - & - \\
\hline 152 & Mimusops elengi & Sapotaceae & - & - & - & - & - & + & - \\
\hline 153 & Mitragyna parvifolia & Rubiaceae & + & - & + & - & - & - & - \\
\hline 154 & Morinda citrifolia & Rubiaceae & + & - & - & - & - & + & - \\
\hline 155 & Morinda pubescens & Rubiaceae & + & - & - & - & - & - & - \\
\hline 156 & Murraya paniculata & Rutaceae & + & - & - & - & - & + & - \\
\hline 157 & Naringi crenulata & Rutaceae & + & + & + & - & - & - & - \\
\hline 158 & Neocinnamomum caudatum & Lauraceae & - & - & + & - & - & - & - \\
\hline 159 & Neolamarckia cadamba & Rubiaceae & + & - & - & - & - & - & - \\
\hline 160 & Nothopegia beyneana & Anacardiaceae & - & - & - & - & - & - & - \\
\hline 161 & Nyctanthes arbor-tristis & Oleaceae & - & - & + & - & - & - & - \\
\hline 162 & Ochna obtusata & Ochnaceae & + & - & - & - & - & - & - \\
\hline 163 & Ocotea lancifolia & Lauraceae & - & - & + & - & - & - & - \\
\hline 164 & Oroxylum indicum & Bignoniaceae & + & - & + & - & - & - & - \\
\hline 165 & Pavetta crassicaulis & Rubiaceae & - & - & - & - & - & - & - \\
\hline 166 & Persea villosa & Lauraceae & - & - & - & - & - & - & - \\
\hline 167 & Phyllanthus emblica & Phyllanthaceae & + & + & - & - & - & - & - \\
\hline 168 & Pittosporum wightii & Pittosporaceae & - & - & - & - & - & - & - \\
\hline 169 & Plumeria rubra & Apocynaceae & - & - & - & - & - & - & - \\
\hline 170 & Polyalthia cerasoides & Annonaceae & - & - & - & - & - & - & - \\
\hline 171 & Polyalthia suberosa & Annonaceae & + & - & + & - & - & - & - \\
\hline 172 & Polyanthia simiarum & Annonaceae & - & - & - & - & - & - & - \\
\hline 173 & Pongamia pinnata & Leguminosae & + & - & + & - & - & + & - \\
\hline 174 & Premna barbata & Lamiaceae & - & - & - & - & - & - & - \\
\hline 175 & Premna mollissima & Lamiaceae & + & - & - & - & - & - & - \\
\hline 176 & Proebe wightii & Lauraceae & - & - & - & - & - & - & - \\
\hline 177 & Protium serratum & Burseraceae & - & - & - & - & - & - & - \\
\hline 178 & Prunus ceylanica & Rosaceae & + & - & + & + & - & - & - \\
\hline 179 & Prunus pygeoides & Rosaceae & - & - & - & - & - & - & - \\
\hline 180 & Psidium guajava & Myrtaceae & - & - & - & - & - & - & - \\
\hline 181 & Psydrax dicoccos & Rubiaceae & + & + & - & - & + & - & - \\
\hline 182 & Pterocarpus marsupium & Leguminosae & + & - & - & - & - & - & - \\
\hline 183 & Pterospermum acerifolium & Malvaceae & - & - & - & - & - & - & - \\
\hline 184 & Pterospermum xylocarpum & Malvaceae & - & - & - & - & - & - & - \\
\hline
\end{tabular}


Mohanta MR et al / Not Sci Biol, 2018, 10(3):354-362

\begin{tabular}{|c|c|c|c|c|c|c|c|c|c|}
\hline 185 & Radermachera xylocarpa & Bignoniaceae & - & - & - & - & - & - & - \\
\hline 186 & Rhus chinensis & Anacardiaceae & - & + & - & - & - & - & - \\
\hline 187 & Salix tetrasperma & Salicaceae & - & + & - & - & + & - & - \\
\hline 188 & Saraca asoca & Leguminosae & + & - & + & - & - & - & - \\
\hline 189 & Schleichera oleosa & Sapindaceae & + & - & + & - & + & - & - \\
\hline 190 & Schrebera swietenioides & Oleaceae & - & - & + & - & - & - & - \\
\hline 191 & Semecarpus anacardium & Anacardiaceae & - & - & - & - & - & + & - \\
\hline 192 & Shorea robusta & Dipterocarpaceae & - & - & - & - & - & - & - \\
\hline 193 & Sloanea sterculiacea & Elaeocarpaceae & - & + & + & - & - & - & - \\
\hline 194 & Soymida febrifuga & Meliaceae & + & - & - & - & - & - & - \\
\hline 195 & Sterculia villosa & Malvaceae & - & - & + & - & - & - & - \\
\hline 196 & Stereospermum chelonoides & Bignoniaceae & + & + & - & - & + & - & - \\
\hline 197 & Stereospermum tetragonum & Bignoniaceae & + & + & - & - & + & - & - \\
\hline 198 & Streblus asper & Moraceae & + & + & + & + & - & - & - \\
\hline 199 & Streblus taxoides & Moraceae & + & + & + & + & - & - & - \\
\hline 200 & Strychnos nux-vomica & Loganiaceae & - & - & - & - & - & - & - \\
\hline 201 & Strychnos potatorum & Loganiaceae & + & - & + & - & - & - & - \\
\hline 202 & Styrax serrulatum & Styracaceae & - & - & + & - & - & - & - \\
\hline 203 & Suregada multiflora & Euphorbiaceae & - & + & + & + & - & - & - \\
\hline 204 & Symplocos cochinchinensis & Symplocaceae & + & + & - & - & - & - & - \\
\hline 205 & Symplocos racemosa & Symplocaceae & - & + & - & - & - & - & - \\
\hline 206 & Syzygium cumini & Myrtaceae & + & - & - & + & + & - & - \\
\hline 207 & Syzygium fruticosum & Myrtaceae & - & - & + & - & - & - & - \\
\hline 208 & Syzygium jambos & Myrtaceae & - & - & - & - & - & + & - \\
\hline 209 & Syzygium nervosum & Myrtaceae & - & - & - & - & - & - & - \\
\hline 210 & Syzygium praecox & Myrtaceae & - & - & - & - & - & - & - \\
\hline 211 & Tamarindus indica & Leguminosae & + & - & - & - & + & - & + \\
\hline 212 & Tamilnadia uliginosa & Rubiaceae & + & - & + & - & - & - & - \\
\hline 213 & Tarenna asiatica & Rubiaceae & + & - & - & - & + & - & - \\
\hline 214 & Tectona grandis & Lamiaceae & - & - & - & - & + & - & - \\
\hline 215 & Terminalia arjuna & Combretaceae & + & - & - & - & - & - & - \\
\hline 216 & Terminalia bellirica & Combretaceae & + & - & + & - & - & - & - \\
\hline 217 & Terminalia chebula & Combretaceae & + & - & + & + & + & - & - \\
\hline 218 & Terminalia tomentosa & Combretaceae & + & - & + & - & - & - & - \\
\hline 219 & Toona ciliata & Meliaceae & - & - & + & - & + & + & - \\
\hline 220 & Trema orientalis & Cannabaceae & - & - & - & - & - & - & + \\
\hline 221 & Trevesia palmata & Araliaceae & - & - & - & - & - & - & - \\
\hline 222 & Triadica cochinchinensis & Euphorbiaceae & - & + & + & + & - & - & - \\
\hline 223 & Turpinia cochinchinensis & Staphyleaceae & + & + & + & - & - & - & - \\
\hline 224 & Vitex glabrata & Lamiaceae & - & + & - & + & - & + & - \\
\hline 225 & Vitex leucoxylon & Lamiaceae & + & - & + & - & - & - & - \\
\hline 226 & Vitex peduncularis & Lamiaceae & - & + & + & + & - & - & - \\
\hline 227 & Vitex pinnata & Lamiaceae & + & + & + & - & - & - & - \\
\hline 228 & Walsura trifoliata & Meliaceae & + & - & - & - & - & - & - \\
\hline 229 & Wendlandia gamblei & Rubiaceae & - & - & - & - & - & - & - \\
\hline 230 & Wendlandia tinctoria & Rubiaceae & + & - & + & - & + & - & - \\
\hline 231 & Wrightia arborea & Apocynaceae & + & + & - & + & - & - & - \\
\hline 232 & Wrightia tinctoria & Apocynaceae & - & - & + & - & - & - & - \\
\hline 233 & Xantolis tomentosa & Sapotaceae & + & - & + & - & - & - & - \\
\hline 234 & Xylia xylocarpa & Leguminosae & - & - & + & - & + & - & - \\
\hline 235 & Xylosma longifolia & Salicaceae & - & - & - & - & - & - & - \\
\hline 236 & Zanthoxylum rhetsa & Rutaceae & + & - & + & + & - & - & - \\
\hline 237 & Ziziphus rugosa & Rhamnaceae & + & - & + & - & - & - & - \\
\hline 238 & Ziziphus glabrata & Rhamnaceae & - & - & - & - & - & - & - \\
\hline 239 & Ziziphus jujuba & Rhamnaceae & + & - & - & - & - & - & - \\
\hline 240 & Ziziphus xylopyrus & Rhamnaceae & + & - & - & - & - & - & - \\
\hline
\end{tabular}

${ }^{*} \mathrm{~A}=$ Sri- Lanka, $\mathrm{B}=$ China, $\mathrm{C}=$ Myanmar, $\mathrm{D}=$ Thailand, $\mathrm{E}=$ Malaysia, $\mathrm{F}=$ Australia, $\mathrm{G}=$ Africa 
360

Environmental barriers and geographical distance are the major factor. Albizia lebbeck and Tremna orientalis are the plants with only Indo-African affinity and Barringtonia acutangula, Lepisanthes rubiginosa, Mimusops elengi, Semicarpus anacardium and Syzygium jambos have only Indo-Australian affinity. There are 20 tree species like Alphonsea lutea, Gardenia lotifolia, Cordia monoica etc. are found to have only Indo-Lankan affinity. Among all SBR trees, there are 38 species found similar between Sri- Lanka and china, 35 species among China and Myanmar and 39 among Sri- Lanka and Myanmar.

Affinity with Sri-Lanka

Tree species of SBR show highest affinity $(46.66 \%)$ with Sri- Lanka (Table 2). Out of 240 tree species of Similipal Biosphere Reserve, 112 species find the distribution in SriLanka. Noteworthy tree species are Morinda citrifolia, Grewiaeriocarpa, Premna mollissima, Vitex pinnata, Stereospermum chelonoides, Benkara fasciculata, Tarenna asiatica, Cordia dichotoma, Symplocos cochinchinensis, Syzygium cumini and Tamarindus indica etc.

Affinity with Myanmar

SBR also exhibits very strong affinity to Myanmar having 110 tree species $(45.83 \%)$ similar to both the region. Some of the common species are Turpinia cochichinensis, Sterculia villosa, Mallotus nudiflorum, Mallotus philippensis, Meliosoma simplicifolia, Meyna spinosa, Mitragyna parvifolia, Naringi crenulata, Ocotea lancifolia, Oroxylum indicum, Saraca asoca, Streblus asper, etc.

\section{Affinity with China}

There are 60 tree species found common to the China and of the study area which is $25 \%$ of the total tree species of
SBR. The tree species like Cassine glauca, Streblus taxoides, Streblus asper, Rhus chinensis, Psydrax dicoccos, Wrightia arborea, Symplocos cochinchinensis, Suregada multifolia, Abizia chinensis, Ficus hispida, Ficus microcarpa, Salix tetrasperma, Falconeria insignis, Cassine glauca etc. are wildly distributed species in both the areas.

Affinity with Thailand

A total of 30 tree species is found to be distributed in both Thailand and the study area. The affinity percentage is $12.5 \%$. The common tree species are Dillenia indica, Albizia chinensis, Xylosma longifolia, Garcinia cowa, Carallia branchiate, Streblus taxoides, Dillenia indica, zanthoxylum rhetsa, Wrightia arborea, Triadica cochinchinensis, Terminalia chebula, Syzygium cumini, Vitex glabrata, Vitex peduncularis etc.

\section{Affinity with Malaysia}

Like Thailand, Malaysia shows $12.5 \%$ affinity but its floristic distribution is different. It has 30 tree species similar to SBR which are Albizia chinensis, Albizia odoratissima, Albizia procera, Alstonia scholaris, Butea monosperma, Chionanthus ramiflorus, Diospyros malabarica, Elacocarpus tectorius, Lanneacoro mandelica, Litsea glutinosa, Micromelum minutum, Schleichera oleosa etc.

\section{Affinity with Australia}

Australia has only 18 tree species similar to SBR with affinity 7.5\%. The tree species namely Morinda citrifolia, Vitex glabrata, Antidesma ghaesembilla, Casearia tomentosa, Flueggea virosa, Mallotus philippensis, Mimusops elengi, Pongamia pinnata, Toona ciliata, Carallia branchiata, Syzygium jambos etc. are distributed in both the areas.
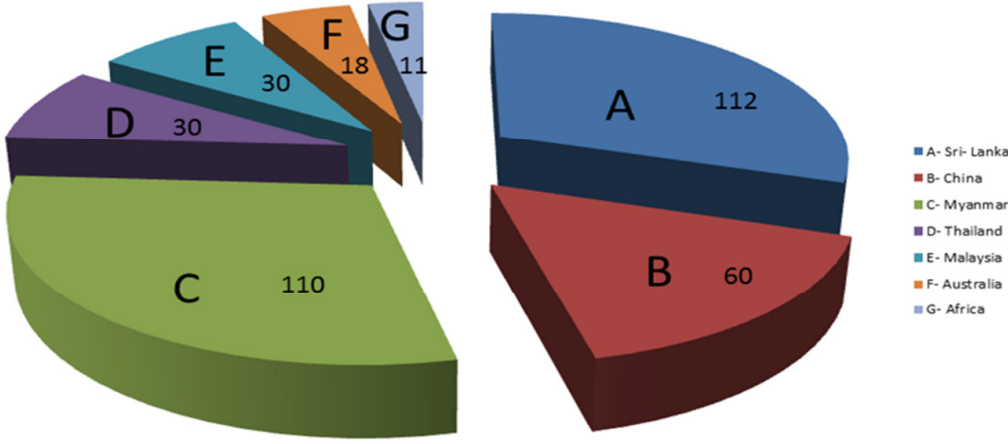

Fig. 2. Phytogeographical affinities of SBR

Table 2. Analytical result of affinities of different countries with SBR tree species

\begin{tabular}{cccc}
\hline S1. No. & Country & No. of species & Percentage $(\%)$ \\
\hline 1 & Sri-Lanka & 112 & 46.66 \\
2 & Myanmar & 110 & 45.83 \\
3 & China & 60 & 25.00 \\
4 & Thailand & 30 & 12.5 \\
5 & Malaysia & 30 & 12.5 \\
6 & Australia & 18 & 7.5 \\
\hline 7 & Africa & 11 & 4.58 \\
\hline
\end{tabular}

\footnotetext{
${ }^{*}$ Percentage is calculated out of 240 tree species of SBR
} 
Affinity with Africa

Affinity of tree species of SBR with African subContinent is poorly represented. Only 11 species are found common between the areas which is $4.58 \%$ of total tree species of SBR. Some of the representative species are Cordia dichotoma, Flacourtia indica, Alangium salviifolium, Crateva marmelos, Alstonia scholaris, Catunaregam spinosa, Ficus benjamina, Flueggea virosa, Grewia tilifolia, Tamarindus indica and Trema orientalis.

\section{Himalayan plants in SBR}

SBR possesses several numbers of tree species and orchids similar to Himalaya. Among them the noteworthy plant species are Psychotria denticulata, Rubia cordifolia, Albizia chinensis, Baccaurea ramiflora, Ficus semicordata, Phoebe lanceolata and Phoebe wightii. Two endemic orchids are also found in SBR namely Cirrhopetalum panigrabinum and Eria meghasaniensis (Misra, 2004).

Different anthropogenic forces cause destruction of the forests of tropical regions worldwide. Eastern Ghats of India is under such anthropogenic stress like cattle grazing, collection of fuel wood and non-timber forest products, illegal extraction of timber, soil mining etc (Muthuramkumar et al., 2014). So there is an urgent need for develop effective conservation strategies to maintain the species diversity in the world. Phytogeographical study is important in conservation aspect because it reflects the status of floral distribution in the world. In the present study, the tree species of Similipal biosphere reserve showed significant affinity with other subcontinents. Sri- Lanka has the highest similarity on tree species distribution with SBR. Muthuramkumar et al. (2014) has also described the affinity of Eastern Ghats trees and found highest affinity with Sri-Lanka. So migration of flora to/from Similipal Biosphere Reserve is not restricted within Indian subcontinent but it is throughout the globe. Neighbouring countries like Sri-Lanka, China, Bangladesh, Pakistan etc. shares several numbers of species. The migration of species is highly influenced by different ecological barrier and environmental conditions. The various postulations such as Pleistocene relicts, Hora's hypothesis on migration of floras from Himalayas to South Indian Hills etc. are yet to establish the phytogeographic characteristics of Similipal Biosphere Reserve.

\section{Conclusions}

Inventory and assessment of floristic composition of an area are prerequisites for planning and implementation of conservation strategies. The origin, distribution and ecological significance of the taxa extend in the area are vital for protected area network. More ever, it will add to biogeographical significance of the locality. The phytogeographical affinity of SBR supports the migration, establishment and naturalization of flora from/to SBR. This hypothesis needs further study for biogeographical mapping of Indian sub-continent.

\section{Acknowledgements}

The present research received no specific grant from any funding agency in the public, commercial, or not-for-profit sectors.

\section{References}

Biswal AK, Nair MM, Upadhyay HS (2011). Flora and fauna of Similipal Biosphere Reserve. Floral Diversity, Vol I. Similipal Biosphere Reserve, Baripada, Odisha.

Brahmam M, Dhal NK, SaxenaHO (1996). Ethnobotanical studies among the Tanla of Malyagiri hills in Dhenkanal district, Orissa, India. In: Jain, SK (Ed). Ethnobiology in Human Welfare. Deep Publications pp 393396.

Collen B, Ram M, Zamin T, Mcrae I (2008). The tropical biodiversity data gap: addressing disparity in global monitoring. Tropical Conservation Science 1:75-88.

Daniel P, Nair NC (1986). The flora of Western Ghats, Review, Proceedings of the Indian Academy ofScience pp 127-163.

Espejel I (1987). A phytogeographical analysis of coastal vegetation in the Yucatan Peninsula. Journal of Biogeography 14(6):499-519.

Gamble JS (1892). The fern of Panchmarhi and those of Mahendragiri. The Indian Forester 18(2):56-57.

Gentry AH (1982). Neotropical floristic diversity: Phytogeographical connections between central and south America, Pleistocene climatic fluctuations, or an accident of the Andean Orodogeny? Annals of the Missouri Botanical Garden 69:557-593.

Ghazanfar SA (1991). Vegetation structure and phytogeography of Jabal Shams, an arid mountain in Oman. Journal of Biogeography 18:299309.

Hains HH (1921-1925). The Botany of Bihar and Orissa, Part 5. London, Adlard and West Newman.

Hooker JD (1904). Pioneer Plant Geography, Royal Botanic Garden, Kew, Surrey.

Hora SL (1949). Satpura hypothesis of the distribution of Malayan fauna and flora of peninsular India. Proceedings of National Institute of Science of India 15(8):309-314.

Hubbard RNLB, Boulter MC (2000). Phytogeography and paleoecology in Western Europe and Eastern Greenland near the Triassic-Jurassic boundary. Palaios 15:120-131.

Kirkpatrick JB (1982). Phytogeographical analysis of Tasmanian alpine floras. Journal of Biogeography 9:255-271.

Krings A (2000). A phytogeographical characterization of the vine flora of the Sonoran and Chihuahuan deserts. Journal of Biogeography 27:1311-1319.

Lausi D, Nimis PL (1985). Quantitative phytogeography of the Yukon Territory (NW Canada) on a chorological phytosociological basis. Vegetation 59:9-20.

Manrique CE, Duran R, Argaez J (2003). Phytogeographic analysis of taxa endemic to the Yucatan Peninsula using geographic information systems, the Domain Heuristic method and Parsimony analysis of endemicity. Diversity and Distributions 9:313-330.

Misra S (2004). Orchids of Odisha. Bishen Singh and Mahendra Pal Singh Publication, Dehradun, India.

Moony HF (1950). Supplement to the botany of Bihar and Orissa, Ranchi, India.

Muthuramkumar S, Ayyappan N, Parthasarathy N, Mudappa D, Raman TRS Selwyn MA, Pragasan LA (2006). Plant community structure in 
362

tropical rain forest fragments of the Western Ghats, India. Biotropica 38:143-160.

Nayak AK, Kar T,Mandal KK(2014). Trees ofSimilipal Biosphere Reserve, Bariapada, Odisha.

Nimis PL, Fonda G (1997). Phytogeography of parasteppic vegetation in the high Friulian plain (NE Italy). Plant Ecology 132:15-28.

Rana TS, Datt B, Rao RR (2001). Vegetational diversity in Tons valley, Garhwal Himalaya (Uttaranchal) India with special reference to phytogeographical. Taiwania 46(3):217-231.

Razi BA (1955). Some aspects of the embryology of Zeylanidium olivaceum and Lawiazeylanica. Bulletin of the Botanical Society of Bengal 9:36-41.

Rzedowski J (1975). An ecological and phytogeographical analysis of the grasslands of Mexico. Taxon 24:67-80.

Sagar R, Raghubanshi AS, Singh JS (2003). Asymptotic models of speciesarea curve for measuring diversity of dry tropical forest tree species Current Sciences 84:1555-1560.

Saravanan S, Ravichandran K, Balasubramanian A, Paneerselvam K (2013). Structureand floristic composition of tree diversity in Andaman tropical evergreen forest of middle Andaman, India. Indian Journal of Forestry 36:167-171.
Saxena HO, Brahmam M (19941996). Flora of Orissa, Vol I-IV. Orissa Forest Development Corporation Ltd. Odisha, India.

Saxena HO, Brahmam M (1989).The flora of Similipahar (Similipal), Orissa. Regional Research Laboratory. Bhubaneswar.

Singh NP, Mudgal V, Khanna KK, Srivastava, Sahoo SC, Bandopadhyay AK, Aziz S, Das M, Bhattacharya RP, Hajra PK (2001). Flora of Bihar. Botanical survey of India. Ministry of Environment and Forests. India.

Sukumar R, Dattaraja HS, Suresh HS, Radhakrishnan J, Vasudeva R, Nirmala S, Joshi NV (1992). Long-term monitoring of vegetation in a tropical deciduous forest in Mudumalai, southern India. Current Science 62:608-616.

Suresh HS, Sukumar R (1999). Phytogeographical affinity of flora of Nilgiri Biosphere Reserve. Rheedea 9(1):1-21.

Takhtajan A (1969). Flowering plants, origin and dispersal. Oliver and Boyd Ltd, Edinburgh pp 236.

Verma DM, Balakrishnan NP, Dixit RD (1993). Flora of Madhya Pradesh. Botanical survey of India. Ministry of Environment and Forests. 